\title{
INTERACTION OF PLANETARY NEBULAE WITH PRENEBULAE DEBRIS
}

\author{
JULIETA FIERRO \\ Instituto de Astronomia, Unam
}

In the present poster we suggest that some of the structures observed in the envelopes of planetary nebulae are caused by the interaction of central star wind and radiation with preplanetary nebula debris: planets, moons, minor objects, ring and ring arcs.

Recently considerable amount of planetary material has been reported to exist around solar type stars, this debris could be evaporated during the envelope ejection and alter the chemical abundance and produce some of the envelope inhomogenities.

If there are massive enough rings of material surrounding the progenitor and planets in their vicinity arc rings could be formed. If the rings are viewed pole on when the envelope is detached from the central star it will interact with the arc ring material and produce "ansae" and pedal and garden hose shape structures observed in some planetaries. 